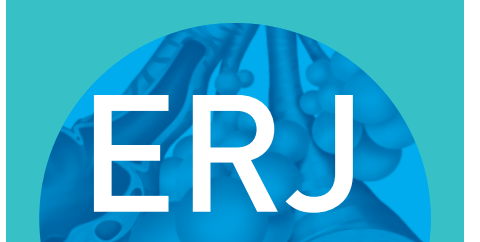

open research
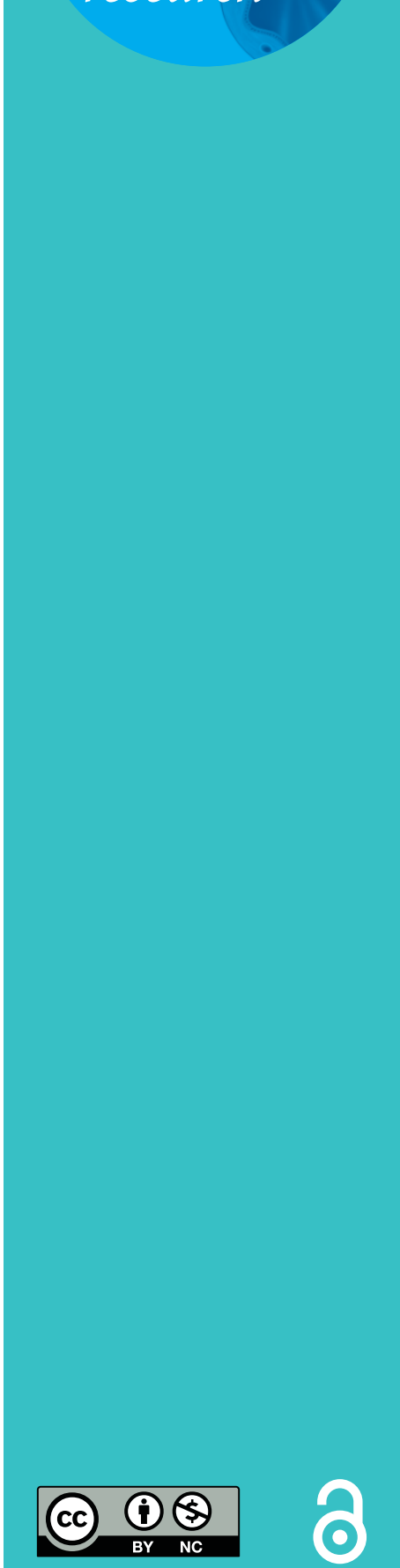

\section{Respiratory care in myotubular myopathy}

\author{
Hui-leng $\operatorname{Tan}^{1}$ and Elaine Chan ${ }^{2}$
}

Affiliations: ${ }^{1}$ Royal Brompton and Harefield NHS Foundation Trust, London, UK. ${ }^{2}$ Great Ormond Street Hospital for Children NHS Foundation Trust, London, UK.

Correspondence: Hui-leng Tan, Royal Brompton Hospital, Sydney Street, London, SW3 6NP.

E-mail: h.tanarbht.nhs.uk

ABSTRACT X-linked myotubular myopathy is a neuromuscular condition caused by pathogenic variants in the MTM1 gene, which encodes for myotubularin, a phosphatidylinositol 3-phosphate phosphatase. Affected individuals typically require intensive medical intervention to survive, though there are some milder phenotypes. To date, respiratory management has been primarily supportive, optimising clearance of airway secretions, providing ventilatory support and prevention/early intervention of respiratory infections. Encouragingly, there has been significant progress in the development of novel therapeutic strategies such as gene therapy, enzyme replacement therapy and drugs that modulate downstream pathways. In this review, we discuss the common respiratory issues using four illustrative real-life cases, and summarise recent translational research, which offers hope to many patients and their families.

$@$ ERSpublications

A proactive approach is the cornerstone to respiratory care in MTM patients: optimising clearance of airway secretions, preventing/early treatment of respiratory infections and providing ventilatory support to those who need it https://bit.ly/2LYFzW2

Cite this article as: Tan H-I, Chan E. Respiratory care in myotubular myopathy. ERJ Open Res 2021; 7: 00641-2020 [https://doi.org/10.1183/23120541.00641-2020].

Copyright $\odot$ The authors 2021. This version is distributed under the terms of the Creative Commons Attribution NonCommercial Licence 4.0. For commercial reproduction rights and permissions contact permissions@ersnet.org 


\section{Introduction}

Centronuclear myopathies derive their name from the characteristic central placement of the myocyte nuclei on muscle biopsy. Historically, myotubular myopathy (MTM) describes the X-linked form, while centronuclear myopathy typically refers to the autosomal dominant and autosomal recessive forms. This review focuses on X-linked MTM, the most common of the three and usually the most severe, requiring the greatest respiratory input. MTM is a neuromuscular condition caused by pathogenic variants in the MTM1 gene located at Xq28 (OMIM 310400). MTM1 encodes the protein myotubularin, a potent phosphatidylinositol 3-phosphate (PI3P) phosphatase, required for myocyte differentiation [1, 2]. Indeed, affected individuals' myocytes display structural features characteristic of fetal myotubes, the basis for its nomenclature, in keeping with premature differentiation arrest or delay at a step preceding myofibre formation [3], though defective structural maintenance has also been postulated [4].

The reported incidence of MTM is estimated to be 1 in 50000 male births. Typical presentation is in the neonatal period with marked weakness, hypotonia, external ophthalmoplegia and respiratory failure [5]. Antenatally, there may be a history of reduced fetal movements, and polyhydramnios [6]. Affected infants are often long for gestational age (birth length $>90$ th centile) and have a large head circumference, which may or may not be associated with hydrocephalus. They typically have narrow, elongated facies and long slender digits [7]. They may also be at increased risk of subdural haemorrhage [8]. Thinning of the ribs on chest radiography has been reported [9]. Previously, most infants did not survive past their first few months of life; however, with intensive respiratory support, many are now surviving into their teenage years or older.

As with all orphan diseases, where the data pool is limited, most information is often derived from descriptive case series. Therefore, in this review, we start by discussing four real-life cases, using them to highlight pertinent points for the subsequent discussion. We then summarise the latest research, including recent multicentre retrospective studies of MTM disease manifestations and medical management, as well as new therapies in the pipeline.

\section{Cases}

Case 1

Patient 1 is now 11 years old. He was born at $36+6$ weeks gestation by elective caesarean section for breech presentation and polyhydramnios. His Apgars were 2 at $1 \mathrm{~min}, 2$ at $5 \mathrm{~min}$ and 5 at $10 \mathrm{~min}$ and he needed intubation and ventilation. He was extubated onto continuous positive airway pressure (CPAP) on day 7 and weaned to nasal cannula oxygen on day 14 . He was noted to have facial weakness, ptosis with limited external ocular movements, generalised hypotonia, poor head and neck control and absent deep tendon reflexes. He required frequent suctioning to manage his oral secretions due to bulbar weakness. Subsequent muscle biopsy suggested centronuclear myopathy with genetics testing confirming MTM. He had a deterioration in his respiratory condition. Chest radiography showed changes in the right middle and upper lobes consistent with aspiration. He was treated with intravenous antibiotics and established on noninvasive ventilation (NIV). Initially, he required $24 \mathrm{~h}$ NIV, but as he improved, he only required NIV when asleep. A $\mathrm{pH}$ study showed severe gastro-oesophageal reflux disease (GORD), a gastrostomy was inserted and Nissen's fundoplication was performed.

Aged 3 months, he had a respiratory arrest with an episode of profound hypoxaemia requiring bag and mask ventilation but not intubation. Sputum and blood cultures were all negative, and in the absence of any rise in inflammatory markers the episode was postulated to have been secondary to mucus plugging. Seven weeks later, he had another respiratory deterioration requiring intubation and transfer to the paediatric intensive care unit (PICU). This exacerbation was preceded by a coryzal prodrome, with increased work of breathing and oxygen requirement. He was finally successfully extubated on the third attempt onto $24 \mathrm{~h}$ NIV. Regular suctioning and intensive chest physiotherapy were needed. He had persistent subsegmental right upper lobe collapse despite bronchoscopic attempts at reinflation. He slowly improved, but remained prone to acute respiratory deteriorations secondary to secretions plugging off his airways, requiring periods of bagging and intensive physiotherapy.

He was discharged home to allow the family quality time together over Christmas and managed to spend 4 months at home before being readmitted with left lower lobe (LLL) collapse. An increase in his NIV pressures was required, but at the cost of abdominal distension, which resolved on subsequent reduction of NIV pressures. A month later, he had another respiratory arrest requiring resuscitation at home. Once again, he had LLL collapse and was admitted for intravenous antibiotics and intensive chest physiotherapy. Copious secretions were cleared at bronchoscopy. He had developed midface hypoplasia from prolonged wearing of the NIV mask resulting in poor mask fit and leak.

After careful discussion with his family, a tracheostomy was inserted at 3 years of age and he was discharged home on 24 -h tracheostomy ventilation. He then had a period of respiratory stability and did 
not require any unscheduled hospital admissions for 7 years. His most recent admission was 2 years ago. On presentation to hospital, he was found to have basal atelectasis and a right pneumothorax that required chest drain insertion (figure 1). He required 12 days on PICU before being discharged home.

To optimise clearance of airway secretions, his daily chest physiotherapy regimen includes the use of the cough-assist device. His current medication includes nebulised $0.9 \%$ saline and Salbutamol immediately before physiotherapy three times a day, nebulised colomycin twice daily after physiotherapy, nebulised DNase $1 \mathrm{~h}$ before physiotherapy once daily and nebulised 7\% hypertonic saline p.r.n. when unwell.

Case 2

Patient 2 was born at term by emergency caesarean section with poor Apgar scores ( 1 at 1 min, 2 at 5 min and 7 at $10 \mathrm{~min}$ ) and required ventilation for $24 \mathrm{~h}$, followed by nasal high-flow oxygen therapy for 7 days. Antenatally, decreased fetal movement and polyhydramnios had been noted.

Postnatally, he was noted to be hypotonic, with little head movement, had feeding difficulties and recurrent aspiration. When he was assessed by the neuromuscular team at 1 month of age, he showed significant hypotonia, had marked stridor and limited visual tracking. He was fed via a nasogastric tube.

Relevant investigation results include: 1) Microlaryngobronchoscopy showed laryngomalacia and vocal cord dyskinesia; 2) electromyogram (EMG) (genioglossus) showed single-fibre EMG as prominently abnormal, suggestive of bulbar palsy; 3) Speech and language assessment confirmed unsafe swallow; 4) normal ECG; and 5) normal magnetic resonance imaging (brain). Most crucially, by 8 months of age, muscle biopsy and genetic testing confirmed the diagnosis of MTM.

At 8 months of age, he presented with left-sided pneumonia, requiring CPAP during his hospitalisation (figure 2a). He was discharged self-ventilating in air. He was prescribed repeated courses of antibiotics due to persistent left-sided consolidation. Suspected gastro-oesophageal reflux was treated. He was also started on a continuous feeding regime to minimise reflux aspiration. Secretions were managed with Hyoscine patches. He was commenced on enteral prophylactic antibiotics.

At 11 months, he suffered an acute respiratory deterioration after a 24-h history of cough, fever and increased work of breathing, secondary to rhinovirus-positive bronchiolitis. Chest radiography showed left lung collapse/consolidation (figure $2 \mathrm{~b}$ and $\mathrm{c}$ ). Poor tolerance of CPAP led to intubation and ventilation. Multiple bronchoscopies were performed in attempts to clear secretions and reinflate a persistent collapsed left lung, albeit with little success, though the ventilatory requirement $\left(15 / 5 \mathrm{cmH}_{2} \mathrm{O}\right)$ was not considered excessive. Following two failed extubations, a respiratory arrest and growing concern regarding reversibility of left lung collapse, serial multidisciplinary team (MDT) meetings were convened to consider the suitability for long-term ventilation via tracheostomy. Eventually, after much debate, the decision to support long-term invasive home ventilation was made and a tracheostomy was inserted (aged 13 months) during the PICU admission. He was discharged from PICU after a 4-month stay.
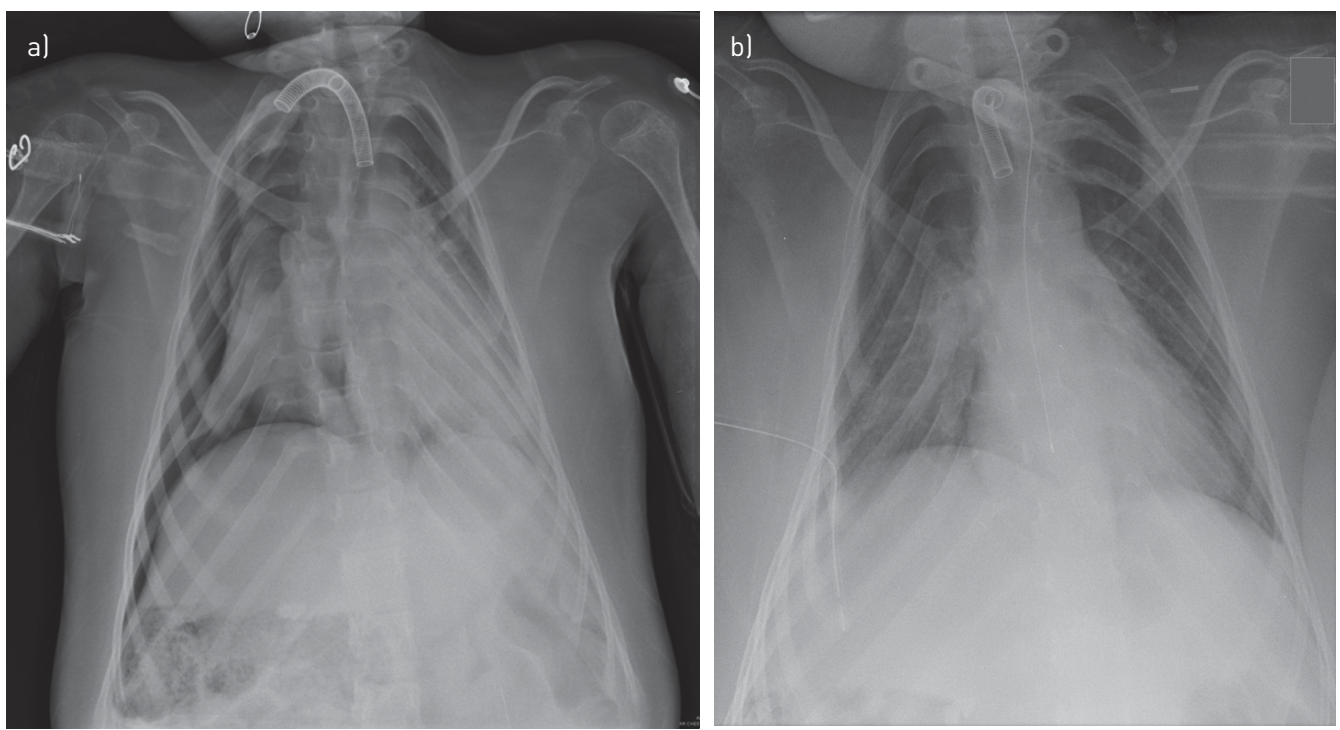

FIGURE 1 Basal atelectasis and right pneumothorax before (a) and after (b) chest drain insertion (Patient 1). 

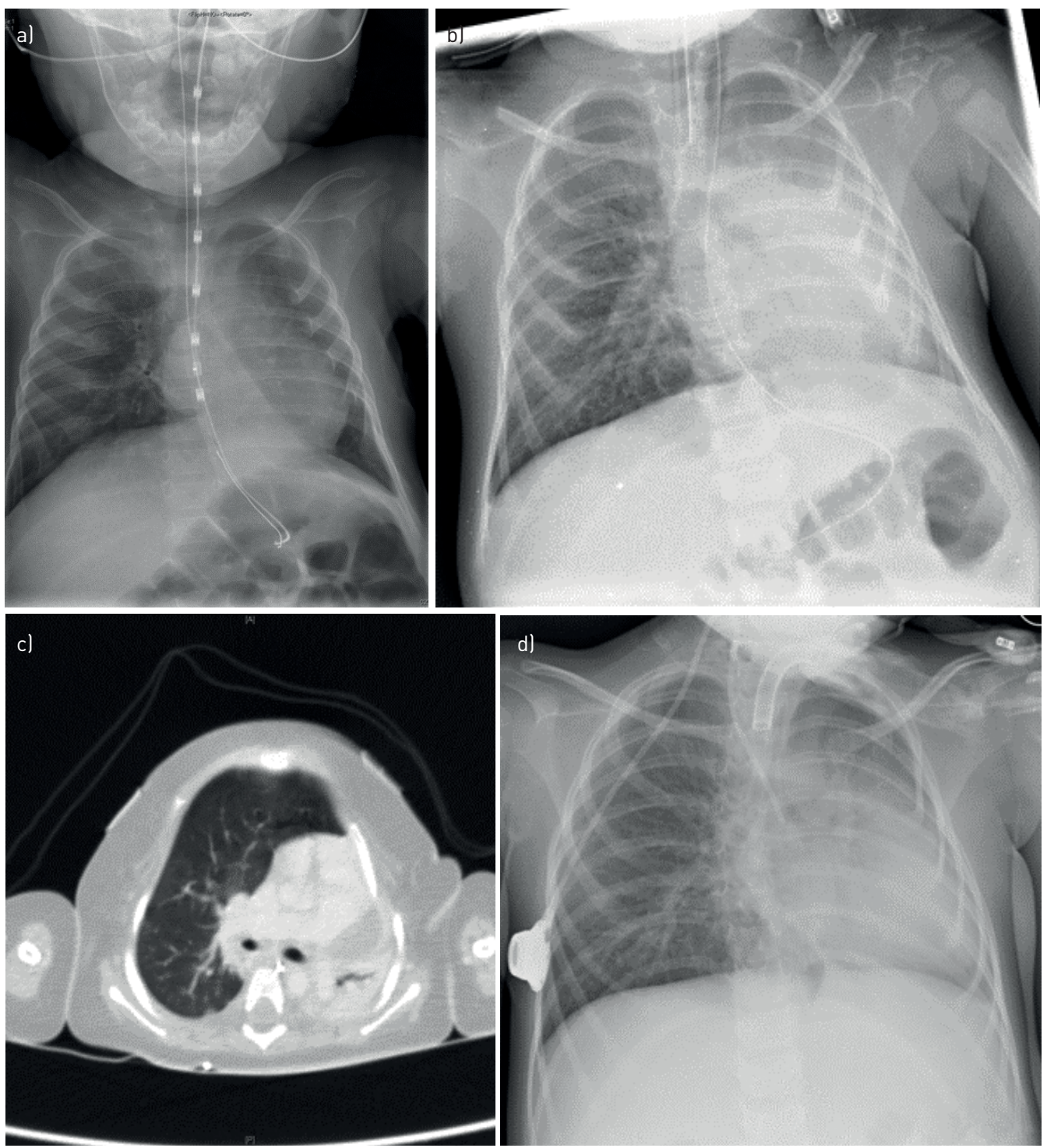

FIGURE 2 a) Chest radiography of Patient 2 at 8 months during an episode of pneumonia demonstrating left lower lobe collapse; b) chest radiography of Patient 2 at 11 months during an episode of pneumonia requiring paediatric intensive care unit admission; c) chest computed tomography of Patient 2 during an episode of pneumonia at 12 months of age; d) second chest radiography of Patient 2 at 26 months of age on discharge from transitional care unit.

During his 12-month stay in the transitional care unit, he had a further respiratory exacerbation, involving left-sided lung collapse with effusion (at 23 months of age) requiring a further PICU admission (including high-frequency oscillation ventilation), where he spent 6 weeks. His airway was colonised by Pseudomonas aeruginosa. By the time he was discharged at 26 months of age, he was ventilated $24 \mathrm{~h} \cdot \mathrm{day}^{-1}$ at pressures of $16 / 7 \mathrm{cmH}_{2} \mathrm{O}$ (figure $2 \mathrm{~d}$ ).

Since discharge, he remains on $24 \mathrm{~h} \cdot \mathrm{day}^{-1}$ tracheostomy ventilation. His secretion management remains challenging, requiring frequent suctioning and regular respiratory physiotherapy (twice daily using the cough-assist device). Other routine respiratory care includes prophylactic antibiotics and the influenza vaccine every winter. He remains nil p.o. and fed only via gastrostomy. Neurologically, he is able to sit with support, reach out and transfer objects with his hands despite hypotonic upper limbs and can stand in his standing frame for $1-2 \mathrm{~h} \cdot \mathrm{day}^{-1}$. He has no verbal communication but can use some sign language.

\section{Case 3}

Patient 3 is the second child of nonconsanguineous parents, whose eldest son also has MTM. There was an antenatal history of polyhydramnios. Genetic testing confirmed he had the same genetic mutation as 
his older brother, but clinically his symptoms were much more severe. His management plan was discussed between his parents and the multidisciplinary team (including neonatologist/neurologist/ respiratory paediatrician) soon after birth. Without any potential curative or disease-modifying treatment available in the horizon at the time, his parents did not wish to pursue long-term invasive ventilation for him and elected for palliative NIV, supported by the palliative care, secondary and tertiary medical and local community teams. In addition, with quality of life being the primary goal of his care, various aspects of an advanced healthcare directive were discussed with his parents in MDT meetings. Besides the form of respiratory support, the consensus view included the use of oral/nasogastric antibiotics only for respiratory infections, nonescalation to active cardiorespiratory resuscitation and paediatric intensive care. He passed away following a respiratory illness at home at the age of 6 months. It is worth mentioning that his older sibling with MTM was on nocturnal NIV, was a wheelchair user and fully integrated into school.

\section{Case 4}

Patient 4 was 11 months old when he presented with respiratory failure secondary to respiratory syncytial virus infection requiring ventilation in PICU. His apparent weakness prompted a neuromuscular work-up, including a muscle biopsy showing nonspecific myopathy. Years later, his mother met a cousin, whose son had a neuromuscular condition, and subsequently family history emerged, leading to MTM1 testing, which came back positive. He was later started on noninvasive ventilation at 8 years of age. He has since been transitioned to the adult service.

\section{Case discussion}

A proactive approach is the cornerstone to respiratory care in patients with MTM. Being aware of potential respiratory issues is crucial so that problems can be anticipated and prevented, or identified and treated early. Regular monitoring with reviews by a MDT, including a respiratory physician, respiratory physiotherapist, speech and language therapist, dietitian, occupational therapist and neuromuscular paediatrician should be part of routine clinical care. The effective mobilisation and clearance of secretions is one of the key pillars in respiratory care. This may include the use of mechanical insufflation/ exsufflation (MI-E) (cough-assist device), which should be considered in children who are very weak, those who cannot cooperate with manual cough-assist or air-stacking or in whom these methods are not effective, and also in those with bulbar dysfunction [10]. The use of MI-E does not remove the need for oral or nasopharyngeal suction.

Assistance with ventilation to optimise gas exchange and prevent atelectasis is normally in the form of bilevel positive pressure ventilation with a backup rate, administered in the form of NIV or invasive ventilation via a tracheostomy. If ventilated, regular monitoring with overnight sleep studies along with analysis of data downloaded from the ventilator, is necessary to ensure ventilatory settings remain optimal with the growth and development of the child. The level of sleep study (e.g. oxycapnography, respiratory polygraphy or polysomnography) would depend on local availability, but the ability to monitor carbon dioxide is essential. Patients with a milder phenotype who do not require ventilatory support, should advisably be screened for sleep-disordered breathing (SDB) if they have a forced vital capacity (FVC) $<60 \%$ predicted, are nonambulant or have any SDB symptoms as recommended by the British Thoracic Guidelines for respiratory management of children with neuromuscular weakness [10].

Children can have recurrent episodes of mucus plugging and be very unstable from a respiratory point of view in their first few years of life. Their respiratory status often then stabilises as they grow older. It is important for children to receive immunisations, such as the yearly influenza vaccine and the pneumococcal vaccine to minimise any respiratory infections [11]. Airway secretions often increase with respiratory viral illnesses, contributing to the development of mucus plugging and atelectasis. Furthermore, viral respiratory infections can predispose to a secondary bacterial infection. When respiratory infections do occur, early treatment with intensifying of chest physiotherapy and, where appropriate, the judicious use of antibiotics is recommended.

Management of oral secretions can be a problem secondary to the orofacial and bulbar weakness of these patients [11]. Anticholinergic medication such as Hyoscine patches and glycopyrronium bromide can be helpful, though should be used with care, starting at a low dose and slowly titrating to an effective dose for the child, while being mindful not to cause excessive thickening of secretions that could inadvertently result in difficulty with clearing airway secretions.

An exploratory study on the use of nebulised saline in children with neuromuscular disease showed it was associated with a decrease in hospitalisations and courses of antibiotics for respiratory infections [12]. Nebulised normal (0.9\%) saline may be considered in children who have tenacious secretions [10]. Anecdotally, nebulised hypertonic $(3 \%$ or $7 \%$ ) saline has been trialled with good effect in a few patients with MTM, when their secretions have become thick and more difficult to clear during a respiratory infection (as 
seen in Patient 1 and 2). Its use has been extrapolated from conditions such as cystic fibrosis where hypertonic saline has proved to be an effective adjunct to physiotherapy during acute exacerbations and regular use reduced the frequency of pulmonary exacerbations [13]. The mechanism of action is thought to be by enhancing mucociliary clearance and there may also be an immunomodulatory effect [14]. Clinical trials are needed in children with neuromuscular conditions to determine whether it has a similar beneficial effect.

We also urgently need good evidence as to whether nebulised antibiotics such as colomycin are effective at reducing the frequency of lower respiratory tract infections in patients who recurrently isolate organisms such as $P$. aeruginosa from their airway secretions. ECKERLAND et al. [15] studied 20 children with severe neurological impairment such as neuromuscular disorders (NMDs) or cerebral palsy, 9 of whom had tracheostomies in situ, and found that nebulised antibiotics reduced the number of respiratory infections from a median (IQR) of $6.0(4.0-10.0)$ episodes in the year prior to starting, to $2.0(1.0-3.0)$ episodes in the year after starting. It was a small, retrospective questionnaire study and one of the limitations was the potential risk of recall bias. However, its findings are consistent with a small retrospective study of 15 adult patients with NMD, which also demonstrated a reduction in respiratory exacerbations and hospital admissions after commencement of nebulised colomycin [16]. Despite the absence of high-level evidence from prospective randomised trials, the use of nebulised antibiotics in patients with chronic microbial airway colonisation and recurrent respiratory infections may be a potentially beneficial management option.

In patients who have frequent respiratory infections, despite optimal respiratory treatment (as in Patient 2), prophylactic enteral antibiotics such as azithromycin are occasionally used. It is crucial that chest physiotherapy is already fully optimised and any causes/exacerbating factors of recurrent respiratory infections, such as GORD or aspiration, are fully treated before prophylactic antibiotics are considered. It is worth noting that antibiotics have no effect on viral infections, only bacterial ones and there is also concern regarding the potential development of antibiotic resistance.

Medical complications that appear unrelated to the primary myopathy have been described in long-term survivors suggesting expression of the defective protein is not limited to myocytes [17, 18]. Examples include: 1) peliosis hepatis, a rare vascular lesion characterised by the presence of multiple blood-filled, cystic cavities within the liver; 2) two patients with MTM, aged 15 years and 21 years, both with chronic respiratory failure requiring tracheostomy ventilation, were recently reported to have pulmonary blebs that ruptured and required drainage [19]. Interestingly, Patient 1 presented with a pneumothorax on his most recent admission, though no obvious blebs were seen on chest radiography. A better understanding of whether certain MTM1 mutations predispose to these complications would be important so that targeted screening could be performed if found to be the case.

In the case of Patient 2, the persistent left lung collapse, despite aggressive treatment in PICU and its impact on ventilation, cast uncertainties on the effectiveness of long-term home ventilation in improving outcome and quality of life for the child. Proactive respiratory physiotherapy, including use of the cough-assist device, played a significant role in improving and stabilising his condition. The decision on whether to embark on long-term invasive ventilation remains a complex one, and needs to take into consideration the wishes of the family, projected quality of life of the child, achieving adequate medical stability to make home ventilation a feasible option, and overall prognosis. The decision remains a very individualised choice. As illustrated in case 3, long-term invasive ventilation may not be the "right" decision for every child. Palliative care is an equally valid choice for some patients. It is important to take a holistic view, keeping the best interests of the child at the centre of all decision-making. A MDT approach in partnership with the parents is recommended.

On a practical level, the choice between invasive ventilation and NIV is influenced by dependency on respiratory support, difficulties in secretion management, which are often associated with bulbar dysfunction, and clinical phenotype, which is linked to prognosis. In children who are dependent on $24 \mathrm{~h} \cdot \mathrm{day}^{-1}$ ventilation, NIV is generally not appropriate, though can be used in the short term as part of palliative care to relieve symptoms and facilitate discharge home, to enable the child to spend some quality time with their family. Invasive ventilation, while enabling direct-airway toileting, is associated with risks such as bacterial colonisation, tube dislodgement and blockage, all of which carry the risk of morbidity or mortality. The efficacy of NIV has significantly improved over the past few years with better ventilators and increased choices of interfaces. When using NIV, care must be taken to monitor for side effects, such as skin breakdown and midface hypoplasia, which is common following prolonged periods of ventilation. Midface hypoplasia can result in poor mask fit and leak, hampering effective ventilation. Similarly, high ventilatory pressures may result in abdominal distension and may be poorly tolerated. It is imperative to minimise the risk of aspiration, for example, by treating any GORD and avoiding feeds if possible, during the periods on NIV. 
Case 3 also highlights how variation of phenotypes can occur within the same family with the same MTM1 mutation. HofFJAN et al. [20] reported how a patient was only diagnosed aged 65 years following sudden exacerbation of muscle weakness during severe pneumonia. He had previously had minimal symptoms until age 52 years, when he noticed slowly increasing weakness in his arms and difficulty climbing stairs. In contrast, his grandson with the same E404K missense mutation in the MTM1 gene had significant hypotonia, delayed motor milestones and recurrent respiratory infections in his first year of life. This is likely related to genetic modifiers, which are still poorly understood. Unrelated individuals with the same mutation have also been reported to have variable phenotypes [21].

There is an extensive number of different MTM1 mutations. Most truncating (loss-of-function) mutations result in the severe phenotype [22, 23]. McENTAGART et al. [21] found an association between the presence of a nontruncating mutation of the MTM1 gene and the mild phenotype. However, numerous nontruncating mutations were also associated with the severe phenotype and these were not confined to recognised functional domains of the protein. In combination with the aforementioned phenotypic variability of patients with the same mutation, the use of mutation analysis to predict prognosis for individual patients in the early period following diagnosis is currently still limited.

Case 4 emphasises the usefulness of family history in helping to make the diagnosis. It is worthwhile and common practice to revisit nonspecific/undiagnosed cases, even years later. The mild phenotype of patient 4 demonstrates the spectrum of disease seen in MTM, which is discussed further in the next section.

\section{Long-term outcomes/natural history of MTM}

Knowledge about the natural history of any condition is an important prerequisite to evaluating any new therapy. The RECENSUS study, a multicentre chart review of 112 patients, was the preparatory stage of the gene therapy program. The primary objective was to characterise disease manifestations and medical management; the secondary objectives to identify prognostic disease variables and potential outcome measures for therapeutic intervention studies. It revealed $90 \%$ of patients required respiratory support at birth [24]. Sixteen patients spent their entire first year of life in hospital. The time spent in hospital was highest in the first year of life, declined in year 2 and continued to decline through year 5. Nearly half of the patients required 24 -h ventilatory support and $60 \%$ had tracheostomies. The median age of tracheostomy insertion was 3 months. Overall mortality was $44 \%$ (64\% in those $\leqslant 18$ months, $32 \%$ in those $>18$ months). The most common cause of death was respiratory failure $(66.7 \%)$ despite the use of respiratory support, followed by cardiorespiratory arrest (18\%) and liver bleeds associated with hepatic peliosis (10\%) [25]. The median survival for patients with a tracheostomy was 22.8 years (IQR 8.7-30.2). Of the 63 patients where such data were available, life-sustaining support in the form of ventilatory support was withdrawn or withheld in 27 (43\%). The median survival for patients receiving life-sustaining care was 19.4 years (IQR 3.1-not estimable) compared with 0.2 years (IQR 0.1-2.1) when life-sustaining support was withdrawn/withheld [25]. There was a wide distribution of MTM1 mutations: 63 patients (43.4\%) had predicted MTM1 null mutations, classified as loss-of-function mutations as they were expected to produce little or no functional or stable myotubularin. The prognosis of this group was significantly worse with median survival of 6.2 years compared with partial loss-of-function patients, who had markedly longer median survival of 30.2 years. The high disease burden and healthcare utilisation reported is in keeping with previous studies $[21,26]$. Of the 33 patients $>18$ months old who had had cognitive assessments, 6 (18\%) were reported as "above grade level," 22 (67\%) were "at grade level," 9 (27\%) were "below grade level," and 3 (9\%) were "significantly below grade level" [24].

In comparison, a prospective longitudinal natural history study of 45 patients from 7 countries was recently published comprising 13, 7 and 25 patients with mild (no ventilatory support), intermediate $(<12 \mathrm{~h}$ ventilatory support per day) and severe ( $\geqslant 12 \mathrm{~h}$ ventilatory support per day) phenotype respectively [27]. In contrast to most other studies that report a higher percentage of patients having the severe phenotype $[26,28]$, this study was skewed towards moderate and mild patients, the authors describe theirs as a natural history study of patients surviving beyond the neonatal period, as neonatal intensive care units were not designated recruitment sites for their cohort. Nonetheless, it gives important insights into the condition. Respiratory distress was present in $91 \%$ of patients at birth and $82 \%$ required respiratory support leading to long hospital stays during the first year of life. Median length of hospital stay was 22, 75, and 142 days for the mild, intermediate and severe groups, respectively. Independent breathing occurred in 31 out of 45 patients and was secondarily lost in 9 patients in the severe group at the median age of 6 months. Of those able to perform pulmonary function tests (PFTs), all, even those with a mild phenotype, had abnormal PFTs with FVC, forced expiratory volume in $1 \mathrm{~s}\left(\mathrm{FEV}_{1}\right), \mathrm{MEP}, \mathrm{MIP}$, and PCF $<80 \%$ predicted. Eighty-nine percent of patients had feeding difficulties and $76 \%$ required feeding tubes.

Three patients died during the 1-year follow-up period, two in the severe category from cardiorespiratory arrest and one in the intermediate category from probable sepsis. Lung function was stable over the 1-year 
period and the most reproducible tests were FVC and MEP. One patient from the mild category started night-time respiratory support a year after enrolment, and one from the severe category changed from invasive to noninvasive ventilation. The degree of respiratory support did not change appreciably in the remaining patients. In terms of motor function, the Motor Function Measure 32 total score decreased by $2 \%$. Overall, regardless of phenotype, MTM is a slowly progressive condition. Similarly, in their cross-sectional online survey and prospective 1 -year phone survey in which $75 \%$ of patients required $>16 \mathrm{~h}$ of respiratory support per day, AmbURGey et al. [26] observed that despite the high burden of disease, the disease course was more stable than they had expected, with few adverse events reported during the course of the year and a lower than expected mortality rate of around $10 \%$ per year.

\section{Research}

Therapeutic strategies which are currently under investigation include gene therapy, enzyme replacement therapy, dynamin-2 modulation and $\mathrm{PIK} 3 \mathrm{C} 2 \mathrm{~B}$ inhibition.

\section{Gene therapy}

Gene therapy using a single dose of recombinant adeno-associated virus (AAV) serotype 8 (AAV8) vector expressing MTM1 administered systemically, has been shown to prolong survival and restore function in both murine and canine models of MTM [29, 30]. Of note, the pathology could be corrected in severely affected mice, implying that the damage induced by the absence of myotubularin is reversible. In contrast to muscular dystrophies such as Duchenne muscular dystrophy, the muscles in MTM are not associated with inflammation nor with the cycles of degeneration/regeneration with resultant replacement with fat and fibrous tissue, suggesting patients in their teens or older could still benefit from gene therapy. There were also no inflammatory, humoral or cytotoxic immune responses against the vector or myotubularin. Myotubularin expression significantly below that of normal levels was sufficient to achieve therapeutic benefit. Of the dogs who received gene therapy, 2 were followed up long term for 4 years. Encouragingly, despite there being a decline in viral copy number and muscle strength over time, their gait, respiratory performance, neurological function and pathology remained comparable to their healthy littermate controls [31]. Another concern had been whether patients might have levels of neutralising antibodies to the vector rendering gene therapy ineffective. Annoussamy et al. [27] reported 10 out of 38 patients had detectable levels of neutralising antibodies to AAV8. Two of the patients, aged 1.7 and 50.8 years, had high inhibitory levels (i.e. an inhibitory titre $>1: 10$ ), potentially rendering AAV8-based gene therapy ineffective. It is anticipated that younger patients would have lower seroprevalence values.

The ASPIRO clinical trial, a multinational, open-label study to evaluate the safety and efficacy of gene therapy using AT132, an AAV8 vector containing a functional copy of the human MTM1 gene, in patients $<5$ years old started in 2017. Participants receive a single dose of AT132 with 5-year follow-up for safety and efficacy. Initial results presented at the 24th International Annual Congress of the World Muscle Society in 2019 were promising: six patients in Cohort 1 had been treated with $1 \times 10^{14}$ vector genomes per kilogram $\left(\mathrm{vg}^{\mathrm{kg}} \mathrm{kg}^{-1}\right.$ ) and four in Cohort 2 had been treated with $3 \times 10^{14} \mathrm{vg} \cdot \mathrm{kg}^{-1}$. The first seven patients treated (all six treated patients in Cohort 1 and the first patient treated in Cohort 2) had achieved ventilator independence. There had also been improvement in neuromuscular function whereby they had achieved the ability to rise to a standing position, or walk. At the time of presentation of the preliminary data, the safety profile appeared manageable with one serious adverse event reported of joint swelling in a patient from Cohort 2, which resolved without treatment. However, since then, 3 patients out of the 17 who received the higher dose developed hepatobiliary toxicity, of whom 2 died from sepsis and 1 from gastrointestinal bleeding. The trial has been paused and an investigation is underway to determine the extent to which AT132 may have contributed to their deaths. The precise mechanisms remain to be elucidated, but some have hypothesised that antibodies to AAV that either pre-exist or develop following vector infusion, may have played a role in the observed toxicity, whereby the formation of vector-antibody complexes could have activated complement through the classic pathway or activated innate immunity through Fc-dependent uptake in antigen-presenting cells [32].

\section{Enzyme replacement therapy}

An alternative strategy to replace myotubularin is to use an intracellular delivery technology. An example is $3 \mathrm{E} 10 \mathrm{Fv}$, a single-chain fragment derived from a mouse monoclonal antibody that enters cells through an equilibrative nucleoside transporter 2, which is expressed at high levels in skeletal muscle. Initial proof-of-concept studies of this enzyme replacement therapy in mice with MTM has shown improvement in muscle contractile function and pathology [33].

\section{Dynamin-2 modulation}

Dynamin 2 modulation may be another promising avenue to pursue. Muscle biopsies from patients with MTM have shown overexpression of dynamin 2 [34]. Interestingly, the autosomal dominant form of 
centronuclear myopathy is caused by mutations in the dynamin 2 gene DNM2 (OMIM \#160150). Some of the DNM2 mutations have been shown to increase dynamin oligomer stability and GTPase activity [35]. Dynamins are GTPase proteins involved in membrane trafficking and endocytosis and in actin cytoskeleton assembly. The injection of dynamin 2 antisense oligonucleotides (ASOs) into Mtm1 knockout mice reduced dynamin 2 protein levels in muscle and prevented the myopathy from developing [36]. Injection into severely affected mice led to reversal of muscle pathology within a fortnight. Another strategy to reduce dynamin 2 levels by using a single intramuscular injection of AAV-short hairpin Dnm2, restored muscle force, mass and histology in Mtm1 knockout mice [37]. A phase I/II clinical trial of DYN101, a synthetically manufactured ASO directed against DNM2 pre-mRNA, started recruiting patients with mutations in MTM1 or DNM2 for the treatment of myotubular and centronuclear myopathies respectively, at the beginning of 2020 .

\section{PIK3C2B inhibition}

Accumulation of PI3P in muscle has been demonstrated in animal models of MTM $[38,39]$ leading to the hypothesis that lowering PI3P levels might prevent or reverse the disease process. Indeed, the PI3kinase inhibitor wortmannin improved motor function and prolonged survival of Mtm1 knockout mice [40].

While the aforementioned therapies are new and thus great care needs to be taken to study their safety profile, tamoxifen, a drug already in clinical use to treat breast cancer, has shown promising results. Two research groups have independently shown that daily oral tamoxifen can improve survival in mice with MTM, while improving overall motor function and preventing disease progression [41, 42]. Its mechanism of action is likely due to its effects on normalising the expression level of the disease modifiers DNM2 and PI3KC2B. Tamoxifen is already known to be safe and well tolerated in children, and clinical trials to see whether these findings in mice translate to clinical benefit in patients should be a priority.

\section{Conclusion}

MTM is a condition associated with significant disease burden, healthcare utilisation and sadly, high mortality, primarily due to respiratory failure. Informed decision-making is crucial when deciding between the different supportive and palliative care options and the "right" choice is different for each child and their family. At the dawning of a new decade, we are poised on the cusp of discovering/developing several new disease-modifying therapies. Results from clinical trials are awaited and will take time, but they offer real hope for the future.

\section{Summary of key points}

- A proactive approach is the cornerstone to respiratory care in patients with MTM.

- The majority of patients will require respiratory support to ensure adequate ventilation and prevent lung atelectasis.

- Effective airway clearance is key in respiratory management. MI-E should be considered in children who 1) are very weak, or 2) have bulbar dysfunction, or 3) cannot cooperate with manual cough-assist or air-stacking, or (4) in whom these aforementioned methods are not effective.

- Nebulised normal and/or hypertonic saline may be considered in children who have tenacious secretions.

- The yearly influenza vaccination is an essential part of basic respiratory care.

- Both unsafe swallow and GORD are very common in children with myotubular myopathy. Early investigation, serial assessments (if needed) and management with insertion of a feeding gastrostomy and Nissen's fundoplication if required, to minimise lung damage from recurrent aspiration, is crucial.

- When respiratory infections do occur, early aggressive treatment with intensifying chest physiotherapy and judicious use of antibiotics where appropriate, are recommended.

- A trial of nebulised colomycin may be considered if the child recurrently grows $P$. aeruginosa from airway secretions and suffers from recurrent chest infections.

- As in many neuromuscular conditions, physical and cognitive capacity are unrelated. Parents often feel that the intellectual capacity of patients with MTM is underestimated due to the severity of their physical disabilities.

- There is a spectrum of severity seen in the condition, and variability can occur even within the same family.

- The decision on whether to embark on long-term invasive ventilation via tracheostomy is a complex one. Factors to be considered include the wishes of the family, (projected) quality of life for the child, achieving adequate medical stability to make home ventilation a feasible option, and overall prognosis. Every child is unique and the decision needs to be made on an individual basis, there is no single "right" decision for all children with MTM. An MDT approach in partnership with parents is recommended. 
Acknowledgements: We are very grateful to the patients and their families who have kindly given permission for us to share their stories at the Respiratory Care in Myotubular Myopathy Symposium and for them to be summarised for this article. We are also grateful to the Myotubular Trust for supporting the Respiratory Care in Myotubular Myopathy Symposium.

Conflict of interest: H-1. Tan has nothing to disclose. E. Chan has nothing to disclose.

\section{References}

1 Taylor GS, Maehama T, Dixon JE. Myotubularin, a protein tyrosine phosphatase mutated in myotubular myopathy, dephosphorylates the lipid second messenger, phosphatidylinositol 3-phosphate. Proc Natl Acad Sci USA 2000; 97: 8910-8915.

2 Blondeau F, Laporte J, Bodin S, et al. Myotubularin, a phosphatase deficient in myotubular myopathy, acts on phosphatidylinositol 3-kinase and phosphatidylinositol 3-phosphate pathway. Hum Mol Genet 2000; 9: 2223-2229.

3 Spiro AJ, Shy GM, Gonatas NK. Myotubular myopathy. Persistence of fetal muscle in an adolescent boy. Arch Neurol 1966; 14: 1-14.

4 Hamanaka K, Inami I, Wada T, et al. Muscle from a 20-week-old myotubular myopathy fetus is not myotubular. Neuromuscul Disord 2016; 26: 234-235.

5 Jungbluth H, Wallgren-Pettersson C, Laporte J. Centronuclear (myotubular) myopathy. Orphanet J Rare Dis 2008 3: 26.

6 Barth PG, Dubowitz V. X-linked myotubular myopathy--a long-term follow-up study. Eur J Paediatr Neurol 1998 2: $49-56$

7 Joseph M, Pai GS, Holden KR, et al. X-linked myotubular myopathy: clinical observations in ten additional cases. Am J Med Genet 1995; 59: 168-173.

8 Koga H, Miyako K, Suga N, et al. Predisposition to subdural hemorrhage in X-linked myotubular myopathy. Pediatr Neurol 2012; 46: 332-334.

9 Osborne JP, Murphy EG, Hill A. Thin ribs on chest X-ray: a useful sign in the differential diagnosis of the floppy newborn. Dev Med Child Neurol 1983; 25: 343-345.

10 Hull J, Aniapravan R, Chan E, et al. British Thoracic Society guideline for respiratory management of children with neuromuscular weakness. Thorax 2012; 67: Suppl. 1, i1-i40.

11 Wang CH, Dowling JJ, North K, et al. Consensus statement on standard of care for congenital myopathies. J Child Neurol 2012; 27: 363-382.

12 Galaz Souza N, Bush A, Tan H-L. Exploratory study of the effectiveness of nebulised saline in children with neurodisability. Eur Respir J 2020; in press [https://doi.org/10.1183/13993003.01407-2020].

13 Wark P, McDonald VM. Nebulised hypertonic saline for cystic fibrosis. Cochrane Database Syst Rev 2018; 9: CD001506.

14 Wright FL, Gamboni F, Moore EE, et al. Hyperosmolarity invokes distinct anti-inflammatory mechanisms in pulmonary epithelial cells: evidence from signaling and transcription layers. PLoS ONE 2014; 9: e114129.

15 Eckerland M, Bock C, Olivier M, et al. Reducing the frequency of respiratory tract infections in severe neurological disorders by inhaled antibiotics: a retrospective data analysis. ERJ Open Res 2019; 5: 00149-2018.

16 Crescimanno G, Marrone O. The microbiome and secondary lung disease in neuromuscular patients: is it time to change our clinical practice? Respirology 2017; 22: 1035-1036.

17 Herman GE, Finegold M, Zhao W, et al. Medical complications in long-term survivors with X-linked myotubular myopathy. J Pediatr 1999; 134: 206-214.

18 Motoki T, Fukuda M, Nakano T, et al. Fatal hepatic hemorrhage by peliosis hepatis in X-linked myotubular myopathy: a case report. Neuromuscul Disord 2013; 23: 917-921.

19 Graham RJ, Ward E. X-linked myotubular myopathy and pulmonary blebs: not just a muscle disorder. Muscle Nerve 2019; 60: E36-E38.

20 Hoffjan S, Thiels C, Vorgerd M, et al. Extreme phenotypic variability in a German family with X-linked myotubular myopathy associated with E404 K mutation in MTM1. Neuromuscul Disord 2006; 16: 749-753.

21 McEntagart M, Parsons G, Buj-Bello A, et al. Genotype-phenotype correlations in X-linked myotubular myopathy. Neuromuscul Disord 2002; 12: 939-946.

22 Biancalana V, Caron O, Gallati S, et al. Characterisation of mutations in 77 patients with X-linked myotubular myopathy, including a family with a very mild phenotype. Hum Genet 2003; 112: 135-142.

23 Laporte J, Biancalana V, Tanner SM, et al. MTM1 mutations in X-linked myotubular myopathy. Hum Mutat 2000; 15: 393-409.

24 Beggs AH, Byrne BJ, De Chastonay S, et al. A multicenter, retrospective medical record review of X-linked myotubular myopathy: the recensus study. Muscle Nerve 2018; 57: 550-560.

25 Graham RJ, Muntoni F, Hughes I, et al. Mortality and respiratory support in X-linked myotubular myopathy: a RECENSUS retrospective analysis. Arch Dis Child 2020; 105: 332-338.

26 Amburgey K, Tsuchiya E, de Chastonay S, et al. A natural history study of X-linked myotubular myopathy. Neurology 2017; 89: 1355-1364.

27 Annoussamy M, Lilien C, Gidaro T, et al. X-linked myotubular myopathy: A prospective international natural history study. Neurology 2019; 92: e1852-e1867.

28 Dowling JJ, Lawlor MW, Das S, et al. X-linked myotubular myopathy. In: Adam MP, Ardinger HH, Pagon RA, Wallace SE, Bean LJH, Stephens K, eds. Gene Reviews. Seattle, University of Washington, 2002.

29 Childers MK, Joubert R, Poulard K, et al. Gene therapy prolongs survival and restores function in murine and canine models of myotubular myopathy. Sci Transl Med 2014; 6: 220ra10.

30 Mack DL, Poulard K, Goddard MA, et al. Systemic AAV8-mediated gene therapy drives whole-body correction of myotubular myopathy in dogs. Mol Ther 2017; 25: 839-854.

31 Elverman M, Goddard MA, Mack D, et al. Long-term effects of systemic gene therapy in a canine model of myotubular myopathy. Muscle Nerve 2017; 56: 943-953.

32 Wilson JM, Flotte TR. Moving forward after two deaths in a gene therapy trial of myotubular myopathy. Hum Gene Ther 2020; 31: 695-696. 
33 Lawlor MW, Armstrong D, Viola MG, et al. Enzyme replacement therapy rescues weakness and improves muscle pathology in mice with X-linked myotubular myopathy. Hum Mol Genet 2013; 22: 1525-1538.

34 Cowling BS, Chevremont T, Prokic I, et al. Reducing dynamin 2 expression rescues X-linked centronuclear myopathy. J Clin Invest 2014; 124: 1350-1363.

35 Wang L, Barylko B, Byers C, et al. Dynamin 2 mutants linked to centronuclear myopathies form abnormally stable polymers. J Biol Chem 2010; 285: 22753-22757.

36 Tasfaout H, Buono S, Guo S, et al. Antisense oligonucleotide-mediated Dnm2 knockdown prevents and reverts myotubular myopathy in mice. Nat Commun 2017; 8: 15661.

37 Tasfaout H, Lionello VM, Kretz C, et al. Single intramuscular injection of AAV-shRNA reduces DNM2 and prevents myotubular myopathy in mice. Mol Ther 2018; 26: 1082-1092.

38 Dowling JJ, Vreede AP, Low SE, et al. Loss of myotubularin function results in T-tubule disorganization in zebrafish and human myotubular myopathy. PLoS Genet 2009; 5: e1000372.

39 Pierson CR, Dulin-Smith AN, Durban AN, et al. Modeling the human MTM1 p.R69C mutation in murine Mtm1 results in exon 4 skipping and a less severe myotubular myopathy phenotype. Hum Mol Genet 2012; 21: 811-825.

40 Sabha N, Volpatti JR, Gonorazky H, et al. PIK3C2B inhibition improves function and prolongs survival in myotubular myopathy animal models. J Clin Invest 2016; 126: 3613-3625.

41 Gayi E, Neff LA, Massana Munoz X, et al. Tamoxifen prolongs survival and alleviates symptoms in mice with fatal X-linked myotubular myopathy. Nat Commun 2018; 9: 4848.

42 Maani N, Sabha N, Rezai K, et al. Tamoxifen therapy in a murine model of myotubular myopathy. Nat Commun 2018; 9: 4849. 